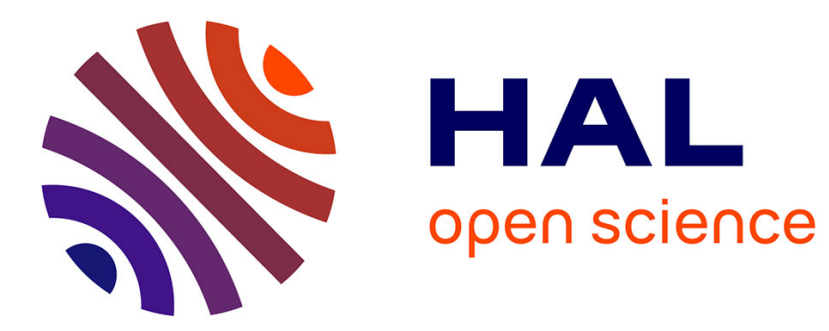

\title{
Echantillonnage des populations aphidiennes en vergers de pêchers. Comparaison de la précision de deux méthodes à l'aide de modèles de simulation d'échantillonnage
}

\author{
Laurent Lapchin, Roger Boll
}

\section{To cite this version:}

Laurent Lapchin, Roger Boll. Echantillonnage des populations aphidiennes en vergers de pêchers. Comparaison de la précision de deux méthodes à l'aide de modèles de simulation d'échantillonnage. Agronomie, 1985, 5 (3), pp.217-226. hal-00884753

\section{HAL Id: hal-00884753 https://hal.science/hal-00884753}

Submitted on 1 Jan 1985

HAL is a multi-disciplinary open access archive for the deposit and dissemination of scientific research documents, whether they are published or not. The documents may come from teaching and research institutions in France or abroad, or from public or private research centers.
L'archive ouverte pluridisciplinaire HAL, est destinée au dépôt et à la diffusion de documents scientifiques de niveau recherche, publiés ou non, émanant des établissements d'enseignement et de recherche français ou étrangers, des laboratoires publics ou privés. 


\title{
Echantillonnage des populations aphidiennes en vergers de pêchers. Comparaison de la précision de deux méthodes à l'aide de modèles de simu- lation d'échantillonnage
}

\author{
Laurent LAPCHIN \\ avec la collaboration technique de Roger BOLL \\ I.N.R.A., Station de Zoologie et de Lutte biologique, 37, bd du Cap, F-06602 Antibes
}

RÉSUMÉ

\begin{abstract}
Deux méthodes d'échantillonnage des pucerons sont comparées en vergers de pêchers : l'une est basée sur la coupe d'un mètre de rameaux par arbre échantillonné, l'autre sur l'examen individuel de l'arbre entier et l'utilisation de classes d'infestation. L'étalonnage de ces classes met en évidence une progression géométrique des effectifs aphidiens.

L'utilisation de modèles de simulation d'échantillonnage permet de comparer la précision des 2 méthodes. Elle dépend essentiellement du degré d'infestation du verger et de l'espèce considérée. La méthode visuelle est géné-
\end{abstract} ralement la plus précise, sauf lorsque la densité est très faible et les espèces peu visibles.

Mots clés additionnels : Myzus persicae, Brachycaudus persicae, Brachycaudus prunicola. simulation models of the sampling procedure.

Two methods of aphid sampling in peach orchards were compared. The first, a branch-cutting method, is based on a one meter length of branch cut per tree. The other consists of a visual observation of the whole tree for 2-4 min according to foliage density, using an infestation scale. When the classes in the scale were calibrated by comparison with the results from the first method a geometrical progression of aphid numbers was noted. The use of models to simulate sampling allowed comparison of the accuracy of the 2 methods. The number of sampled trees in every class was fitted to the POLYA-AEPl'1.1 distribution (ANSc OMBE, 1949). In the branchcutting method, we simulated the distribution of aphid population on a tree, using different distribution models. In the visual method, we constructed 2 probability matrices assigning an infested tree to every visual class (table 5). The coefficients of variation of the 2 sets of results were then fitted to the variation of every parameter (table 4 and 6). For low densities of inconspicuous species, which develop rather small colonies, well distributed in the whole tree, the branch-cutting method was slightly more precise. However, for high densities of conspicuous species whose colonies can reach some size, the visual method clearly showed greater efficiency. It also had the advantage of being paster and non-destructive, and allowing the dynamics of aphid populations on every tree to be followed.

Additional key words : Myzus persicae, Brachycaudus persicae, Brachycaudus prunicola.

\section{INTRODUCTION}

Les études de dynamique des populations aphidiennes sur plantes pérennes, et notamment en vergers, se heurtent à la difficulté de l'échantillonnage. Les techniques utilisées dans le cadre de la "lutte intégrée » sont dérivées des travaux de BAGGiolini (1965) sur le pommier. Elles fournissent généralement une informa- tion qualitative ou semi-quantitative (présence-absence, taux d'organes contaminés...). La quantification des pucerons présents sur un arbre a été abordée par LECLANT \& REMAUDIÈRE (1970) et par TAMAKI (1973) sur pêchers. Les premiers ont estimé les effectifs par arbre d'une espèce, Myzus persicae Sulz., à l'aide de classes d'infestation établies selon les puissances de 5 . Le second a rapporté les comptages des pucerons de la 
même espèce au mètre de rameaux. Toutefois, alors que d'autres travaux ont quantifié la précision de l'échantillonnage dans le cas de cultures annuelles, notamment de céréales (BARIDEAU et al., 1975), la précision des résultats fournis par les méthodes applicables aux plantes pérennes n'a pas été définie.

Nous avons utilisé simultanément, dans 2 vergers de pêchers d'âge différent, une méthode visuelle présentant des points communs avec celle de LECLANT \& REMAUDIĖRE (1970) et une méthode par coupe de rameaux, comparable à celle de TAMAKI (1973). L'écart entre les résultats fournis par les 2 modes d'échantillonnage a ainsi été mesuré. Cet écart dépend d'une série de paramètres susceptibles d'influencer la précision des résultats : caractéristiques de la répartition spatiale de chaque espèce aphidienne dans le verger, entre arbres et à l'intérieur de ceux-ci, caractéristiques des plantes hôtes (quantité de bois) et, dans le cas de la méthode visuelle, efficacité de l'observateur. Le recours à la modélisation permet de simuler des échantillonnages et de déterminer la précision de chacune des 2 méthodes en faisant varier chacun de ces paramètres.

Les 3 espèces aphidiennes les plus représentées dans les vergers expérimentaux ont été étudiées : $M$. persicae, Brachycaudus persicae Pass. et Brachycaudus prunicola Kalt.

\section{LIEU D’ÉTUDE ET MÉTHODES}

\section{A. Lieu d'étude}

Les essais ont été conduits dans 2 vergers expérimentaux situés dans le domaine de la Station d'Amélioration des Plantes de l'I.N.R.A. à Fréjus (Var). Il s'agit de pêchers (cv. «Redhaven », greffés sur franc) âgés respectivement de 13 à 15 ans et de 2 à 4 ans au moment de l'expérimentation. La plantation est espacée de $4 \mathrm{~m}$ en lignes et de $5 \mathrm{~m}$ en colonnes. La conduite de taille est en gobelets différés. Le verger le plus âgé compte environ 270 arbres sur une superficie de $5500 \mathrm{~m}^{2}$, le plus jeune, 130 arbres sur $2600 \mathrm{~m}^{2}$. Les interventions chimiques sont limitées à un traitement d'hiver contre la cloque et à des apports printaniers d'engrais.

Dans chaque verger, une cinquantaine d'arbres échantillons ont été choisis. Dans le verger âgé, ces arbres sont répartis selon une distribution uniforme ( 1 arbre sur 2 dans 1 colonne sur 3 ). Dans le jeune verger, le choix des arbres échantillons a été réalisé après stratification en fonction de critères morphologiques (LAPCHIN \& BOLL, 1981).

\section{B. Echantillonnage par coupe de rameaux}

Une quantité de bois correspondant approximativement à $1 \mathrm{~m}$ de rameaux a été prélevée sur chaque arbre selon une périodicité mensuelle, durant toute la saison de végétation. En 1981, ces prélèvements ont porté sur les arbres échantillons. A partir de 1982, afin de ne pas trop léser les plantes par des prélèvements de bois répétitifs, les coupes de rameaux ont porté sur des arbres hors échantillon. Les prélèvements ont alors été regroupés par verger.
Au laboratoire, les rameaux sont mesurés en séparant le bois d'un an du bois de l'année et les différentes espèces aphidiennes sont récoltées au pinceau. Lorsque les quantités de pucerons sont très importantes, l'échantillon est lavé à l'eau pulvérisée, puis fractionné en quarts ou seizièmes avant le comptage, à l'aide de l'appareil de WATERS (1969), adapté aux échantillonnages de pucerons par RABASSE \& BOUCHERY (1977).

Les effectifs des aphides sont rapportés au mètre de rameaux d'un an en début de saison et au mètre de bois de l'année dès que le rapport bois de l'année sur bois d'un an dépasse 1. La correspondance avec l'effectif par pêcher est obtenue à partir du métrage total de bois d'un an, mesuré chaque hiver sur tous les arbres échantillonnés.

\section{Echantillonnage par classes visuelles}

Toutes les 2 semaines, les arbres échantillons des 2 vergers sont observés pendant 2 à $4 \mathrm{mn}$ chacun, selon la densité du couvert végétal. L'observation se fait à hauteur d'homme ainsi que dans les parties inférieures de la couronne. L'abondance de chaque espèce aphidienne est ensuite notée en fonction de l'échelle suivante (petite colonie : moins d'une trentaine d'individus ; grosse colonie : plus d'une trentaine d'individus) :

Classe 0 : absence

1: 1 petite colonie

2 : plusieurs petites ou une seule grosse colonie

3 : une grosse colonie et une ou plusieurs petites

4 : moins d'une dizaine de grosses colonies

5 : plus d'une dizaine de grosses colonies, réparties dans la couronne de l'arbre

6: une charpentière très infestée et peu de grosses colonies ailleurs

7 : une charpentière très infestée et de nombreuses grosses colonies ailleurs

8 : arbre totalement infesté.

Ces classes correspondent à des degrés d'infestation aisés à distinguer les uns des autres et permettent donc une appréciation rapide. L'observateur ne cherche pas à inspecter chaque rameau et chaque feuille mais à se faire une idée globale de l'infestation.

\section{RÉSULTATS}

Les symboles utilisés dans ce chapitre et leur signification sont rassemblés dans le tableau 1 .

\section{A. Etalonnage des classes de la méthode visuelle}

Les classes visuelles ne correspondant pas à une quantification a priori, il est nécessaire de les étalonner pour chaque espèce et chaque verger expérimental. La démarche la plus logique consisterait à effectuer des coupes de rameaux sur plusieurs arbres de chaque classe visuelle d'infestation, et cela pour chaque espèce aphidienne et sur des vergers d'âge différent. Devant l'impossibilité matérielle d'une telle expérimentation, l'étalonnage a été réalisé en 2 temps : détermination de la forme de la fonction qui relie l'indice visuel à 


\section{TABLEAU 1}

Signification des symboles utilisés.

Meaning of the used symbols.

A Nombre d'arbres échantillonnés.

C Effectif calculé par la méthode de coupe de rameaux.

Co Raison de la progression géométrique qui définit la taille moyenne des colonies en fonction de la classe visuelle de l'arbre.

$\mathrm{Cl}$ Rapport écart-type/moyenne de la loi lognormale qui définit l'effectif d'une colonie.

$\mathrm{K}$ Paramètre de la loi binomiale-négative.

K0 Coefficient de variation des résultats d'échantillonnage de la méthode par coupe de rameaux.

K1 Coefficient de variation des résultats d'échantillonnage de la méthode visuelle.

M0 Métrage moyen de rameaux par arbre.

M1 Ecart-type du métrage de rameaux par arbre.

NO $1^{\text {er }}$ paramètre de la loi de POLYa-AEPPll qui définit le nombre d'arbres par classe visuelle.

N1 $3^{\text {e }}$ paramètre de la loi de POLYA-AEPPLI.

$\mathrm{n}_{\mathrm{V}}$ Nombre d'arbres de la classe visuelle $\mathrm{V}$.

$\mathrm{U}$ Raison de la progression géométrique qui définit l'effectif d'un arbre en fonction de sa classe visuelle.

$\mathrm{V}$ Numéro de la classe visuelle.

l'effectif par arbre ; détermination par espèce et par verger des paramètres de cette fonction.

La $1^{\text {re }}$ partie de ce calcul est basée sur les données de 1981, où les coupes de rameaux ont été effectuées sur les arbres échantillons, et pour l'espèce $B$. persicae (tabl. 2). On observe, dans ce cas, une relation linéaire entre le logarithme des effectifs $\mathrm{C}$, calculés à partir des coupes de rameaux, et le numéro $\mathrm{V}$ de la classe visuelle d'infestation : $\log C=a+b V$.

Le coefficient de corrélation linéaire est de 0,97 pour 7 points et passe à 0,995 si l'on supprime la classe 6 , constituée de 3 arbres seulement. L'effectif de la classe 0 , ne correspondant à aucun aphide observé, devrait théoriquement être nul. En fait, l'observateur qui attribue cette classe à un arbre risque fort de sousestimer la population alors qu'il ne peut pas la surestimer. L'effectif moyen des arbres de la classe 0 n'est donc pas nul. Pour la commodité des calculs, on lui attribue la valeur 1 . Dans ces conditions, «a » est égal à 0 et l'équation précédente devient : $\mathrm{C}=\mathrm{U}^{\mathrm{V}}$.

Les effectifs des classes visuelles successives sont donc sensiblement en progression géométrique de raison $U$ et de $1^{\text {er }}$ terme 1 . La détermination de $U$, par verger et par espèce, constitue la $2^{\mathrm{e}}$ partie de l'étalonnage. A chaque date, l'effectif moyen par arbre est :

$$
\mathrm{N}=\frac{1}{\mathrm{~A}} \sum_{\mathrm{v}=0}^{\mathrm{V}=8} \mathrm{n}_{\mathrm{v}} \cdot \mathrm{U}^{\mathrm{v}}
$$

où $\mathrm{A}$ est le nombre d'arbres échantillonnés et $\mathrm{n}_{\mathrm{V}}$ le nombre de pêchers de la classe $V$ à la date considérée. On dispose ainsi, sur les 3 années, de 6 à 13 dates selon les espèces et les vergers, pour lesquelles les 2 méthodes peuvent être comparées (pour $M$. persicae sur le jeune verger, les données sont trop peu nombreuses pour être utilisées). L'estimation de U est effectuée à l'aide d'un programme d'itérations à partir de la méthode des moindres carrés. Afin de ne pas limiter la valeur de l'estimation aux classes les plus élevées, le calcul est réalisé sur les logarithmes des valeurs observées et estimées. Le tableau 3 présente les valeurs de $\mathrm{U}$ obtenues et l'écart moyen entre les méthodes (sur les logarithmes décimaux), ainsi que la quantification des classes visuelles.

\section{B. Simulation de l'échantillonnage par coupe de rameaux}

\section{Le modèle de simulation d'échantillonnage}

Le modèle simule la coupe de rameaux, situés au hasard dans la couronne de chaque arbre échantillonné. La présence éventuelle d'une ou plusieurs colonies de pucerons sur cette portion de bois ainsi que leurs effectifs sont déterminés par des lois de distribution portant, d'une part, sur la répartition des agrégats entre les arbres échantillonnés ou à l'intérieur de chaque arbre et, d'autre part, sur la dimension des colonies. Le modèle s'appuie donc sur un certain nombre d'hypothèses faites sur ces distributions.

a) La distribution des nombres d'arbres dans les classes visuelles suit une loi de POLYA-AEPPLI (in ANSCOMBE, 1949) ou «Poisson-logarithmique». Cette loi correspond à une répartition au hasard des agrégats avec une distribution géométrique du nombre d'individus par agrégat. Elle est donc en concordance avec les résultats de l'étalonnage des classes visuelles. C'est par ailleurs la loi qui fournit les meilleurs ajustements, pour toutes les dates d'échantillonnage (testés sur les distributions observées de $B$. persicae dans le verger âgé). Afin de diminuer la durée de ces ajuste-

TABLEAU 2

Effectif moyen par arbre de chaque classe visuelle, calculé à partir des coupes de rameaux sur le verger âgé, en 1981. Les nombres d'arbres correspondants sont indiqués entre parenthèses.

Mean number of aphids per tree in every visual class, calculated from cut branches in the old orchard during 1981. (Numbers of sampled trees in brackets.)

\begin{tabular}{|c|c|c|c|c|c|c|c|}
\hline \multirow[t]{2}{*}{ Espèces } & \multicolumn{7}{|c|}{ Classes visuelles d'abondance } \\
\hline & 0 & 1 & 2 & 3 & 4 & 5 & 6 \\
\hline Brachycaudus persicae & $\begin{array}{c}115 \\
(187)\end{array}$ & $\begin{array}{r}591 \\
(34)\end{array}$ & $\begin{array}{r}1972 \\
(29)\end{array}$ & $\begin{array}{r}4294 \\
(25)\end{array}$ & $\begin{array}{r}33286 \\
(29)\end{array}$ & $\begin{array}{r}113872 \\
\text { (9) }\end{array}$ & $\begin{array}{r}69181 \\
\\
\end{array}$ \\
\hline Brachycaudus prunicola & $\begin{array}{c}178 \\
(137)\end{array}$ & $\begin{array}{r}7091 \\
(12)\end{array}$ & $\begin{array}{l}1190 \\
\quad(17)\end{array}$ & $\begin{array}{c}188 \\
(6)\end{array}$ & $\begin{array}{r}8801 \\
\quad(34)\end{array}$ & & \\
\hline Myzus persicae & $\begin{array}{c}519 \\
(131)\end{array}$ & $\begin{array}{l}524 \\
(14)\end{array}$ & $\begin{array}{r}7673 \\
(7)\end{array}$ & $\begin{array}{r}2087 \\
\quad(7)\end{array}$ & & & \\
\hline
\end{tabular}


TABLEAU 3

Etalonnage des indices visuels : raison de la progression géométrique des indices (U) et écart moyen des logarithmes décimaux des effectifs par arbre estimés par les deux méthodes.

Calibration of the visual scale : geometrical ratio of the index (U) and standard error of the decimal logarithm of the numbers, estimated by the two methods.

\begin{tabular}{|c|c|c|c|c|c|c|c|c|c|c|c|}
\hline \multirow{2}{*}{ Espèces } & \multirow{2}{*}{$\mathbf{U}$} & \multirow{2}{*}{$\begin{array}{c}\mathrm{Nb} \\
\text { dates } \\
\text { concer- } \\
\text { nées }\end{array}$} & \multirow{2}{*}{$\begin{array}{l}\text { Ecart } \\
\text { moyen } \\
\left(\log _{10}\right)\end{array}$} & \multicolumn{8}{|c|}{ Effectifs par arbre des classes visuelles } \\
\hline & & & & 0 & 1 & 2 & 3 & 4 & 5 & 6 & 7 \\
\hline \multicolumn{12}{|l|}{ Verger âgé : } \\
\hline Brachycaudus persicae & 6,70 & 13 & 0,61 & 1 & 6,70 & 44,9 & 301 & 2013 & 13484 & 90318 & 604975 \\
\hline Brachycaudus prunicola & 8,34 & 9 & 0,95 & 1 & 8,34 & 69,6 & 581 & 4848 & 40453 & 337550 & 2816621 \\
\hline Myzus persicae & 18,90 & 11 & 0,99 & 1 & 18,90 & 357 & 6756 & 127710 & 2414237 & & \\
\hline \multicolumn{12}{|l|}{ Jeune verger : } \\
\hline Brachycaudus persicae & 6,72 & 7 & 0,64 & 1 & 6,72 & 45,2 & 304 & 2040 & 13711 & 92150 & 619316 \\
\hline Brachycaudus prunicola & 11,24 & 6 & 0,43 & 1 & 11,24 & 126 & 1418 & 15937 & 179059 & 2011843 & \\
\hline
\end{tabular}

ments, les effectifs des différentes classes visuelles ont été réduits par homothétie, en ramenant la valeur de U de 6,7 à 2 . L'ajustement est par ailleurs amélioré en remplaçant l'effectif 1 de la $1^{\text {re }}$ classe par 0 et en décalant les autres valeurs de 2 unités. Dans ces conditions, la correspondance entre classes visuelles et nombres de pucerons par arbre est :

$\begin{array}{llllrrrrr}\text { Classe } & 0 & 1 & 2 & 3 & 4 & 5 & 6 & 7 \\ \text { Effectif } & 0 & 4 & 6 & 10 & 18 & 34 & 66 & 130\end{array}$

Ces modifications améliorent les ajustements mais n'interviennent pas dans le calcul des effectifs de pucerons puisque les paramètres de la loi de distribution ne sont utilisés que pour estimer le mieux possible le nombre d'arbres de chaque classe visuelle. L'effectif " réel » de chacun d'eux est ensuite déterminé à partir des valeurs de $U$. Chaque distribution est définie par 3 paramètres : le nombre A d'arbres échantillonnés, la moyenne N0 de la distribution de POLYA-AEPPLI et son $3^{\mathrm{e}}$ paramètre $\mathrm{N} 1$ (variance/moyenne - 1 ).

b) Le métrage de rameaux par arbre suit, à chaque date, une distribution normale de moyenne Mo et d'écart-type M1 (hypothèse approximativement vérifiée par les données expérimentales).

c) On considère que les colonies sont réparties au hasard dans la couronne des arbres: le nombre de colonies sur $1 \mathrm{~m}$ échantillonné suit alors une loi de Poisson. Cette répartition s'écarte sensiblement des distributions observées sur le terrain. L'agrégation des colonies sur les charpentières est cependant très variable d'une espèce à l'autre et la loi de PoIsSON doit être considérée comme limite. Les conséquences de cette hypothèse sont discutées plus loin.

d) L'effectif moyen par colonie est fonction du degré d'infestation de l'arbre : plus la population est importante et plus les colonies sont grosses. On utilise une progression géométrique pour calculer cet effectif moyen en fonction des classes visuelles, progression dont la raison est $\mathrm{C} 0$. Il s'agit ici d'une hypothèse sommaire, basée sur quelques observations. Le choix d'une progression géométrique permet cependant, selon les valeurs de $\mathrm{C} 0$, de passer d'une indépendance totale entre la taille des colonies et le degré d'infestation des arbres à une gamme de variation importante suivant les classes.

e) Les effectifs des colonies, pour une classe visuelle donnée, sont distribués autour de leur moyenne selon

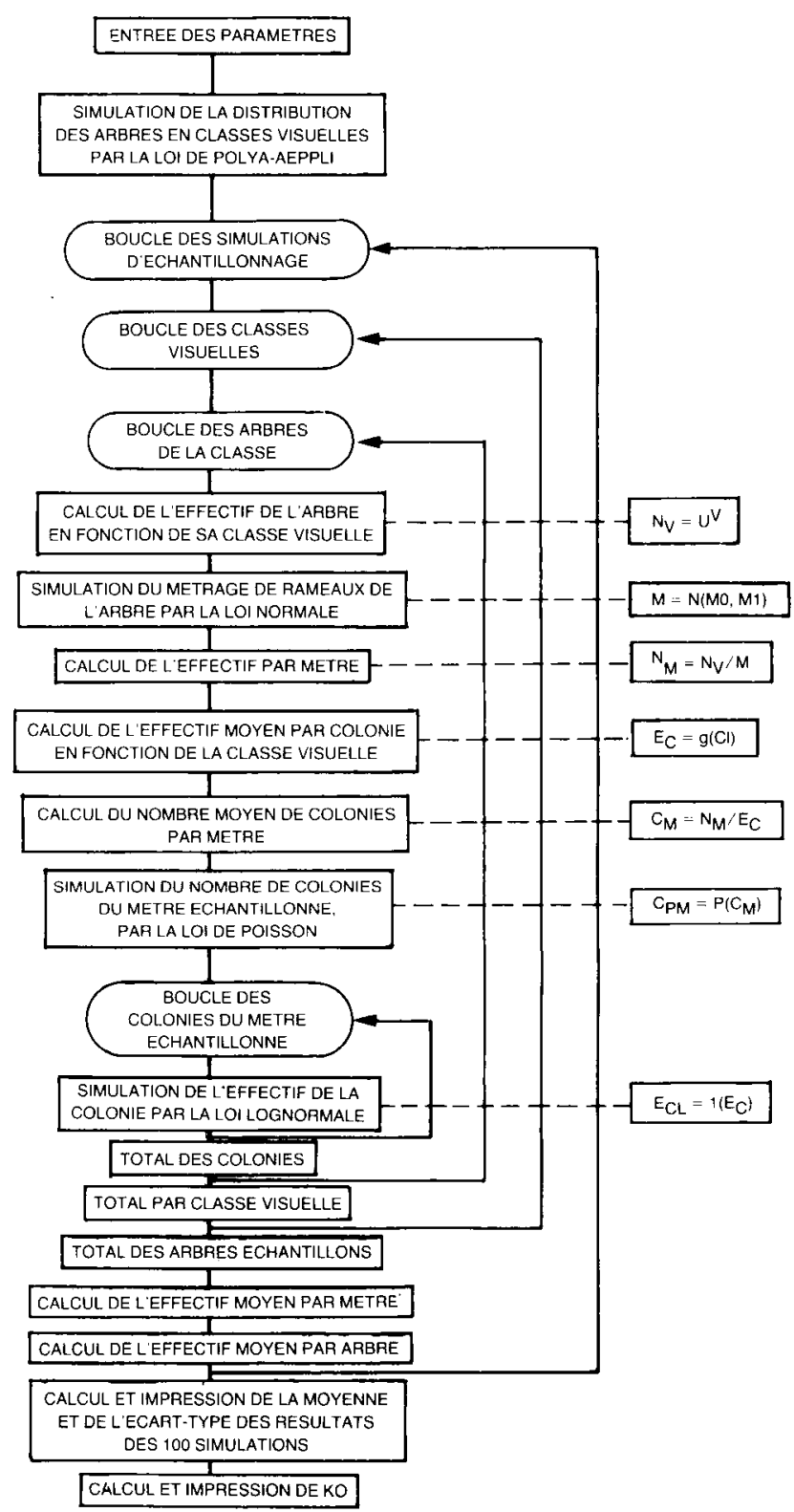

Figure 1

Organigramme du modèle de simulation d'échantillonnage par la méthode de coupe de rameaux.

Flowchart of the simulation model for sampling by the branchcutting method. 
une loi lognormale dont le rapport écart-type sur moyenne est $\mathrm{C} 1$. Ce type de distribution est fréquemment observé dans les colonies aphidiennes, sur différentes cultures.

Le modèle, dont l'organigramme est représenté par la figure 1 , utilise donc 8 paramètres : A, U, N0, N1, $\mathrm{M} 0, \mathrm{M} 1, \mathrm{C} 0$ et $\mathrm{C} 1$. Il détermine d'abord le nombre d'arbres par classe visuelle à partir des paramètres de la loi de POLYA-AEPPLI. Il effectue ensuite 100 simulations d'échantillonnage sur ces arbres pour chaque série de paramètres. On peut alors mesurer la variabilité des résultats et étudier leur distribution.

\section{Distribution des résultats d'échantillonnage}

La distribution de 100 résultats, analysée pour quelques séries de paramètres, s'ajuste très bien avec une loi binomiale négative dont le $3^{\mathrm{e}}$ paramètre $\mathrm{K}$ est défini par : $\mathrm{K}=\mathrm{m}^{2} /\left(\sigma^{2}-\mathrm{m}\right)$.

Ce coefficient permet de comparer les dispersions des résultats pour différentes séries de paramètres : pour une moyenne $\mathrm{m}$ donnée, les résultats sont d'autant plus dispersés que $\mathrm{K}$ est plus petit. Il constitue la meilleure illustration du resserrement des données d'échantillonnage. Dans le cas de la méthode visuelle par contre, les résultats ne s'ajustent pas à une distribution binomiale négative. Afin de comparer les 2 méthodes, c'est le coefficient de variation $\mathrm{K} 0$ des effectifs (rapport écart-type sur moyenne) qui sera pris en compte désormais et non plus le paramètre $K$. Le passage de l'un à l'autre peut se faire de la manière suivante : lorsque le rapport entre la moyenne de la binomiale négative (nombre de pucerons par arbre) et le coefficient $K$ est élevé (en pratique, dès que ce rapport dépasse 2 ou 3), on peut assimiler la distribution à une loi normale. Le coefficient de variation de cette loi normale est alors égal à $1 / \sqrt{\mathrm{K}}$.

\section{Influence des paramètres du modèle sur la distribu- tion des simulations}

Les rôles respectifs des 8 paramètres sont analysés en faisant varier chacun d'eux dans un intervalle plausible, tout en fixant les valeurs prises par les autres. La forme de la fonction qui relie $\mathrm{K} 0$ au paramètre variable est déterminée par ajustement (tabl. 4). Afin de limiter le nombre des combinaisons possibles, les valeurs attribuées aux paramètres fixés n'ont pas été choisies au hasard dans leur intervalle de variation plausible, mais correspondent sensiblement à des situations observées sur le terrain, dans des conditions d'infestation faible, moyenne ou forte.

A noter que la valeur 3,38 attribuée dans certains cas au paramètre $\mathrm{C} 0$ est telle que la dimension des colonies de la classe maximum observée sur le verger âgé (7) soit de 5000 aphides. Par ailleurs, l'ajustement des données de terrain à la loi de POLYA-AEPPI.I montre que le paramètre $\mathrm{N} 0$ est toujours inférieur à N1. Les variations de NO sont donc étudiées dans l'intervalle de $\mathrm{N} 1 / \mathrm{N} 0$ à N1. Enfin, en ce qui concerne le métrage de rameaux, ce n'est pas l'écart-type M1 qui a été fixé mais le coefficient de variation M1/M0.

Les fonctions qui lient les paramètres étudiés aux valeurs de $\mathrm{K} 0$ sont soit linéaires (M1), soit des relations puissances positives $(\mathrm{C} 0, \mathrm{Cl}, \mathrm{M} 0)$ ou négatives (A, U, NO). Aucune fonction simple ne s'adapte aux variations du paramètre $\mathrm{Nl}$.

\section{Simulation de l'échantillonnage par la méthode visuelle}

Pour cette $2^{\mathrm{e}}$ méthode, un modèle de simulation de l'échantillonnage a également été élaboré. La $1^{\text {re }}$ partie du modèle détermine, comme précédemment, le nombre d'arbres échantillons par classe visuelle d'infestation, à partir du nombre A de pêchers examinés et des

TABLEAU 4

Détermination de la fonction qui relie le coefficient de variation KO des résultats d'échantillonnage par la méthode de coupe de rameaux avec les paramètres du modèle. Le paramètre variable est encadré.

Determination of the function connecting the coefficient of variation KO of the branch-cutting results with the parameters. The variable parameter is shown 'boxed'.

\begin{tabular}{|c|c|c|c|c|c|c|c|c|c|c|}
\hline $\mathrm{U}$ & A & No & N1 & $\mathrm{CO}$ & $\mathrm{C} 1$ & Mo & M1 & Formule de régression & $\mathrm{r}$ & $\begin{array}{l}\text { nombre } \\
\text { de points }\end{array}$ \\
\hline 5 à 15 & 53 & 9,698 & 18,95 & 3,38 & 1 & 57 & 10 & $\mathrm{~K} 0=13,20 \quad \mathrm{U}^{-1.69}$ & 0,97 & 11 \\
\hline 5 à 25 & 52 & & & 3,38 & 1 & 57 & 10 & $\mathrm{~K} 0=8,4 \mathrm{I} \mathrm{U}^{-1,17}$ & 0,997 & 21 \\
\hline 6,70 & 10 à 90 & 2,302 & 9,09 & 3,38 & 1 & 57 & 10 & $\mathrm{~K} 0=8,38 \quad \mathrm{~A}^{-0,58}$ & 0,94 & 9 \\
\hline 6,70 & 10 à 90 & 0,692 & 11,69 & 3,38 & 1 & 57 & 10 & $\mathrm{~K} 0=6,80 \quad \mathrm{~A}^{-0,38}$ & 0,82 & 9 \\
\hline 6,70 & 10 à 250 & 14,58 & 16,77 & 3,38 & 1 & 57 & 10 & $\mathrm{~K} 0=1,72 \quad \mathrm{~A}^{-0,37}$ & 0,96 & 26 \\
\hline 6,70 & 50 & 0,5 à 5 & 5 & 3,38 & 1 & 50 & 10 & $\mathrm{~K} 0=1,36 \mathrm{~N} 0^{-0,57}$ & 0,99 & 10 \\
\hline 6,70 & 50 & 1 à 10 & 10 & 3,38 & 1 & 50 & 10 & $\mathrm{~K} 0=1,10 \quad \mathrm{~N} 0^{-0,43}$ & 0,93 & 10 \\
\hline 6,70 & 50 & 1,5 à 15 & 15 & 3,38 & 1 & 50 & 10 & $\mathrm{~K} 0=1,01 \quad \mathrm{~N} 0^{-0,40}$ & 0,91 & 10 \\
\hline 6,70 & 50 & 2 à 20 & 20 & 3,38 & 1 & 50 & 10 & $\mathrm{~K} 0=0,99 \quad \mathrm{~N} 0^{-0,33}$ & 0,95 & 10 \\
\hline 6,70 & 50 & 2,5 à 25 & 25 & 3,38 & 1 & 50 & 10 & $\mathrm{KO}=1,05 \quad \mathrm{~N} 0^{-0,37}$ & 0,91 & 10 \\
\hline 6,70 & 50 & 3 à 30 & 30 & 3,38 & 1 & 50 & 10 & $\mathrm{~K} 0=1,18 \mathrm{~N} 0^{-0,42}$ & 0,98 & 10 \\
\hline 6,70 & 50 & 3,5 à 35 & 35 & 3,38 & 1 & 50 & 10 & $\mathrm{~K} 0=1,43 \mathrm{~N} 0^{-0,50}$ & 0,98 & 10 \\
\hline 6,70 & 53 & 9,698 & 18,951 & 2,2 à 4,4 & 1 & 57 & 10 & $\mathrm{~K} 0=0,025 \mathrm{C}^{2,71}$ & 0,996 & 18 \\
\hline 6,70 & 53 & 9,698 & 18,951 & 3,38 & 0,1 a 13 & 57 & 10 & $\mathrm{~K} 0=0,50 \mathrm{C} 1^{0,09}$ & 0,85 & 38 \\
\hline 6,70 & 53 & 9,698 & 18,951 & 3,38 & 1 & 10 à 310 & $\mathrm{M} 0 / 5$ & $\mathrm{~K} 0=0,10 \mathrm{M} 0^{0,43}$ & 0,97 & 15 \\
\hline 6,70 & 53 & 9,698 & 18,951 & 3,38 & 1 & 57 & 1 à 114 & $\mathrm{~K} 0=0,47+0,008 \mathrm{M} 1$ & 0,98 & 19 \\
\hline
\end{tabular}




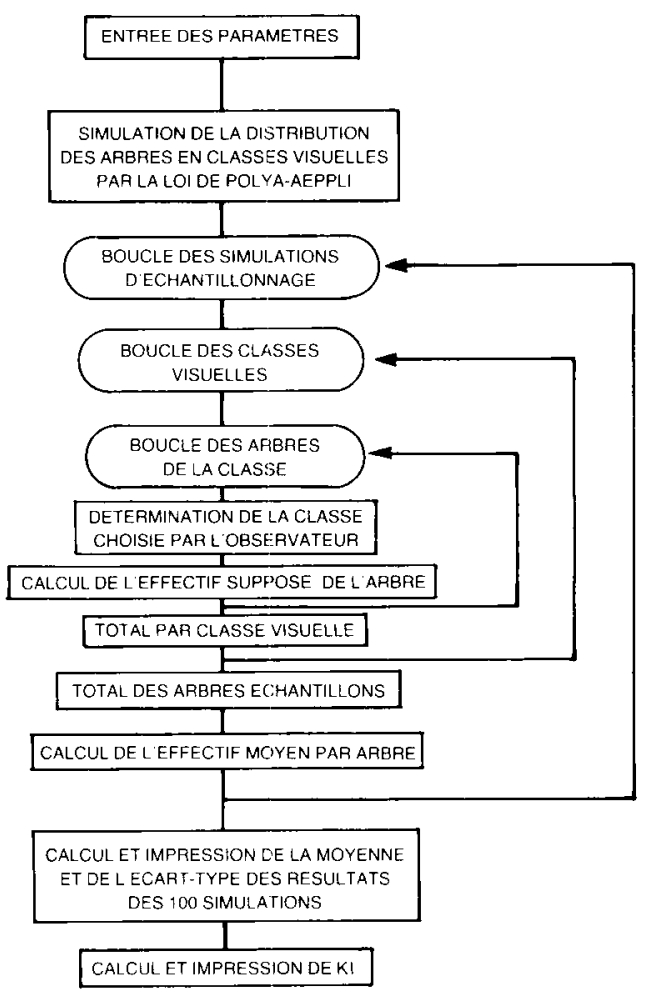

Figure 2

Organigramme du modèle de simulation d'échantillonnage par la méthode visuelle.

Flowchart of the simulation model for sampling by the visual method.

paramètres $\mathrm{N} 0$ et $\mathrm{N} 1$ de la loi de POLYA-AEPPLI. Cette distribution est considérée comme la répartition " réelle » des arbres échantillons dans les classes.

On cherche ensuite dans quelle classe visuelle un observateur situera chacun de ces arbres. Pour une classe « réelle » donnée, on affecte à chaque classe visuelle voisine une probabilité d'être choisie par l'observateur. On obtient ainsi une matrice de probabilités. Cette matrice n'est pas symétrique ; en effet, la probabilité de voir moins de pucerons qu'il n'y en a en réalité est toujours plus forte que la probabilité d'en comptabiliser davantage. La sous-estimation de la classe de l'arbre est donc plus fréquente que sa surestimation. Par ailleurs, le risque de se tromper est généralement plus fort en faible densité qu'en forte densité.

La simulation de l'échantillonnage, dont l'organigramme est représenté par la figure 2 , situe donc chaque arbre de chaque classe " réelle » dans une classe observée, d'après cette matrice de probabilités. L'effectif moyen par arbre est ensuite calculé à partir des effectifs par classe visuelle (déterminés comme précédemment par une progression géométrique de raison U). Comme dans le cas de l'échantillonnage par coupe de rameaux, chaque simulation est répétée 100 fois

Le modèle utilise donc 4 paramètres, $A, N 0, N 1$ et $\mathrm{U}$, ainsi que la matrice de probabilités. Toutefois, compte-tenu du caractère particulier de celle-ci, la distribution des résultats de simulation n'est pas régulière ; on ne peut l'ajuster à une loi simple. L'analyse du rôle des différents paramètres est faite en fonction du coefficient de variation $\mathrm{K} l$ des simulations.

Deux matrices de probabilités, établies d'après l'expérience des observateurs, ont été utilisées : l'une d'erreur « forte », l'autre d'erreur « faible » (tabl. 5). La $1^{\text {re }}$ correspond à une mauvaise visibilité des pucerons et pourrait s'appliquer par exemple à $M$. persicae ; la $2^{\mathrm{e}}$ représente une bonne appréciation de l'infestation et pourrait correspondre à $B$. persicae ou à B. prunicola.

De la même façon que pour la méthode précédente, on fait varier l'un des paramètres en attribuant aux autres des valeurs proches de situations observées, et ceci pour les 2 matrices de probabilités.

Les fonctions qui relient les paramètres étudiés à K1 ne sont pas toujours simples. Il s'agit de fonctions puissances, positive pour $\mathrm{U}$, négative pour $\mathrm{A}$. Les coefficients $\mathrm{N} 0$ et $\mathrm{N} 1$ de la loi de POLYA-AEPPLI qui définit la distribution des arbres en classes visuelles sont liés à $\mathrm{K} 1$ d'une façon moins régulière (fig. 3) : les valeurs de K1 variant linéairement avec N0, pour les valeurs de ce paramètre supérieures à 2 , peuvent être très dispersées pour les faibles valeurs de No. En effet, si la classe 0 est très largement dominante, le risque d'erreur est limité. Par contre, si les classes de faible infestation mais non nulles sont prépondérantes, la variabilité des résultats est nettement plus élevée car ces classes sont toujours entachées d'un risque d'erreur assez fort.

Les matrices de probabilités n'étant pas symétriques, leur impact est difficile à apprécier. Les résultats du tableau 6 montrent que les variations de $\mathrm{Kl}$ en fonction des différents paramètres sont très proches pour les 2 matrices de probabilités testées. La précision de l'échantillonnage dépend donc peu de la matrice d'erreur. La figure 3 permet cependant de constater

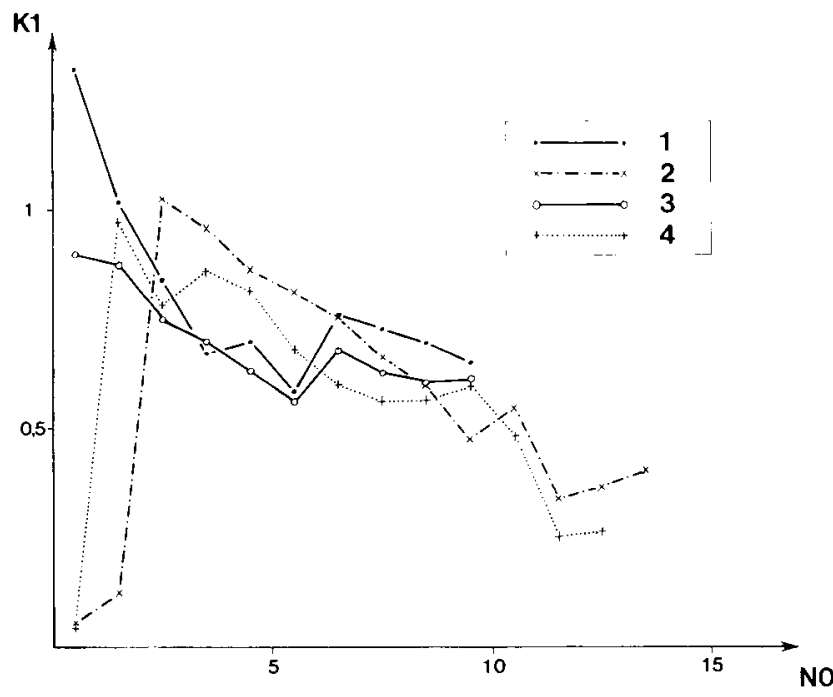

Figure 3

Relation entre le coefficient de variation $K I$ des résultats d'échantillonnage par la méthode visuelle et la movenne No de la distribution des arbres en classes visuelles selon la loi d' POL.YA-At:PPl.I.

1. Matrice d'erreur « forte »; NI $=10$.

2. Matrice d'erreur " forte »; $N 1=20$.

3. Matrice d'erreur « faible »; N1 $=10$.

4. Matrice d'erreur «faible $; N 1=20$.

Relationship between the coefficient of variation $K I$ of the results of the visual sampling method, and the mean NO of tree distribution into visual classes, according to the POL YA-AEPPII distribution.

1. "Sirong » error matrix; $N 1=10$.

2. «Strong " error matrix; $N 1=20$.

3. "Weak» error matrix; $N 1=10$.

4. «Weak» error matrix; $N I=20$. 
TABLEAU 5

Matrices de probabilités d'affectation d'un arbre à une classe visuelle donnée, selon sa classe réelle d'appartenance.

Matrix of probabilities for assigning a tree to a given visual class, according to its real class.

\begin{tabular}{|c|c|c|c|c|c|c|c|c|}
\hline \multicolumn{9}{|c|}{ Matrice de probabilité d'erreur «forte » } \\
\hline \multirow{2}{*}{$\begin{array}{l}\text { Classe attribuée } \\
\text { par l'observateur }\end{array}$} & \multirow[b]{2}{*}{0} & \multirow[b]{2}{*}{1} & \multicolumn{3}{|c|}{ Classes « réelles » } & \multirow[b]{2}{*}{5} & \multirow[b]{2}{*}{6} & \multirow[b]{2}{*}{7} \\
\hline & & & 2 & 3 & 4 & & & \\
\hline 0 & 1,00 & 0,60 & 0,20 & 0,10 & 0,05 & 0 & 0 & 0 \\
\hline 1 & 0 & 0,40 & 0,20 & 0,20 & 0,05 & 0 & 0 & 0 \\
\hline 2 & 0 & 0 & 0,50 & 0,20 & 0,10 & 0 & 0 & 0 \\
\hline 3 & 0 & 0 & 0,10 & 0,40 & 0,20 & 0,05 & 0 & 0 \\
\hline 4 & 0 & 0 & 0 & 0,10 & 0,50 & 0,20 & 0 & 0 \\
\hline 5 & 0 & 0 & 0 & 0 & 0,10 & 0,65 & 0,20 & 0,05 \\
\hline 6 & 0 & 0 & 0 & 0 & 0 & 0,10 & 0,70 & 0,25 \\
\hline 7 & 0 & 0 & 0 & 0 & 0 & 0 & 0,10 & 0,70 \\
\hline
\end{tabular}

\begin{tabular}{|c|c|c|c|c|c|c|c|c|}
\hline \multicolumn{9}{|c|}{ Matrice de probabilité d'erreur « faible » } \\
\hline \multirow{2}{*}{$\begin{array}{l}\text { Classe attribuée } \\
\text { par I'observateur }\end{array}$} & \multicolumn{8}{|c|}{ Classes « réelles » } \\
\hline & 0 & 1 & 2 & 3 & 4 & 5 & 6 & 7 \\
\hline 0 & 1,00 & 0,20 & 0 & 0 & 0 & 0 & 0 & 0 \\
\hline 1 & 0 & 0,80 & 0,10 & 0 & 0 & 0 & 0 & 0 \\
\hline 2 & 0 & 0 & 0,80 & 0,10 & 0 & 0 & 0 & 0 \\
\hline 3 & 0 & 0 & 0,10 & 0,80 & 0,10 & 0 & 0 & 0 \\
\hline 4 & 0 & 0 & 0 & 0,10 & 0,80 & 0,10 & 0 & 0 \\
\hline 5 & 0 & 0 & 0 & 0 & 0,10 & 0,80 & 0,10 & 0 \\
\hline 6 & 0 & 0 & 0 & 0 & 0 & 0,10 & 0,80 & 0,15 \\
\hline 7 & 0 & 0 & 0 & 0 & 0 & 0 & 0,10 & 0,85 \\
\hline
\end{tabular}

TABLEAU 6

Détermination de la fonction qui relie le coefficient de variation KI des résultats d'échantillonnage par la méthode visuelle avec les paramètres du modèle. Le paramètre variable est encadré.

Determination of the function connecting the coefficient of variation $K 1$ of the visual sampling results with the parameters of the model. The variable parameter is shown 'boxed'.

\begin{tabular}{|c|c|c|c|c|c|c|c|}
\hline $\mathrm{U}$ & $\mathrm{N}$ & No & N1 & $\begin{array}{c}\text { Matrice de probabilité } \\
\text { d'erreur }\end{array}$ & $\mathrm{K} 1$ & $r$ & $\begin{array}{l}\text { Nombre } \\
\text { de points }\end{array}$ \\
\hline 3 à 25 & 50 & 1 & 10 & forte & $\mathrm{K} 1=0,312 \cdot \mathrm{U}^{0,687}$ & 0,94 & 23 \\
\hline 3 à 25 & 50 & 1 & 10 & faible & $\mathrm{Kl}=0,201 \cdot \mathrm{U}^{0,779}$ & 0,95 & 23 \\
\hline 3 à 25 & 50 & 7 & 20 & forte & $K 1=0,147 \cdot U^{0,744}$ & 0,96 & 23 \\
\hline 3 à 25 & 50 & 7 & 20 & faible & $\mathrm{K} 1=0,114 \cdot \mathrm{U}^{0,817}$ & 0,98 & 23 \\
\hline 6,70 & 21 à 441 & 3,5 & 10 & forte & $\mathrm{K} 1=5,000 \cdot \mathrm{N}^{-0,508}$ & 0,98 & 21 \\
\hline 6,70 & 21 à 378 & 3,5 & 10 & faible & $\mathrm{K} 1=4,002 . \mathrm{N}^{-0,491}$ & 0,99 & 18 \\
\hline 6,70 & 21 à 462 & 7 & 20 & forte & $\mathrm{K} 1=3,923 \cdot \mathrm{N}^{-0,473}$ & 0,98 & 22 \\
\hline 6,70 & 21 à 420 & 7 & 20 & faible & $\mathrm{K} 1=4,124 . \mathrm{N}^{-0,500}$ & 0,99 & 20 \\
\hline 6,70 & 50 & 2,5 à 9,5 & 10 & forte & $(\mathrm{K} 1=0,771-0,0111 . \mathrm{N} 0)$ & 0,35 & 8 \\
\hline 6,70 & 50 & 2,5 à 9,5 & 10 & faible & $(\mathrm{K} 1=0,741-0,0158 . \mathrm{N} 0)$ & 0,64 & 8 \\
\hline 6,70 & 50 & 2,5 à 13,5 & 20 & forte & $\mathrm{K} 1=1,158-0,0634 . \mathrm{N} 0$ & 0,97 & 12 \\
\hline 6,70 & 50 & 2,5 à 12,5 & 20 & faible & $\mathrm{K} 1=1,011-0,0565 . \mathrm{N} 0$ & 0,94 & 11 \\
\hline
\end{tabular}

que les variations de $\mathrm{K} 1$ deviennent très dépendantes de ces matrices dans le cas d'une très faible infestation (N0 inférieur à 2).

\section{DISCUSSION}

\section{A. Etalonnage des indices visuels}

L'étalonnage des indices visuels met en évidence une progression géométrique de raison $U$ des effectifs aphidiens des classes successives, alors que la définition de celles-ci ne laissait prévoir aucune quantification parti- culière. En fait, il semble que la perception par l'observateur des niveaux d'infestation s'adapte facilement à ce genre de fonction. En effet, les classes de la méthode de LECLANT \& REMAUDIÈRE (1970) suivent également une progression géométrique, de raison 5 . Malgré sa simplicité, la formule qui permet de déterminer l'effectif d'un arbre d'une classe visuelle $\mathrm{V}$ par $\mathrm{U}^{\mathrm{V}}$ fournit donc un moyen fiable de calculer les effectifs de pucerons dans un verger.

Les ordres de grandeur de U pour une espèce donnée ne semblent pas différents sur les 2 vergers suivis dans cette expérimentation. La différence d'âge des arbres, pourtant nobable ici, n'intervient pas d'une façon déci- 
sive dans la quantification des indices. Ce résultat a une grande importance car il permet l'utilisation de la méthode visuelle dans des vergers du même type (même conduite de taille) sans qu'il soit nécessaire de procéder à un nouvel étalonnage. Par ailleurs, les valeurs de U sont voisines pour les 2 espèces de Brachycaudus. Les valeurs légèrement supérieures pour $B$. prunicola proviennent de la dimension des colonies qui sont généralement plus grosses que chez $B$. persicae. Le coefficient élevé de $M$. persicae est dû à la mauvaise visibilité de cette espèce : la sousestimation de l'indice est alors très fréquente et l'étalonnage des classes visuelles compense ce décalage en attribuant une forte valeur à $U$.

\section{B. Fonctionnement des modèles de simulation d'échan- tillonnage}

Les 2 modèles de simulation d'échantillonnage s'appuient sur le résultat de l'étalonnage des indices et supposent que les effectifs suivent une progression géométrique de classe en "classe. Les simulations ne permettent donc pas de remettre en cause ce modèle qui fait la qualité de l'échantillonnage visuel. Toutefois, l'importance des intervalles de variation de U utilisés limite les incertitudes à ce niveau.

Parmi les autres hypothèses constitutives du modèle de l'échantillonnage par coupe de bois, certaines sont peu susceptibles d'influencer les résultats soit parce qu'elles ont peu d'effet sur l'échantillonnage (normalisé de la distribution des métrages de rameaux par arbre), soit parce qu'elles ont peu de chances de s'écarter fortement de- la réalité (distribution de POLYAAEPPLI des arbres dans les classes; distribution lognormale de la dimension des colonies autour de leur moyenne). Par contre, 2 hypothèses peuvent jouer un rôle beaucoup plus net. La répartition au hasard des colonies dans la couronne de l'arbre est rarement vérifiée : on observe généralement une distribution contagieuse dont le degré d'agrégation dépend de l'espèce, du moment par rapport à l'infestation initiale, du degré d'infestation, etc. Le choix d'une répartition selon une loi de POISSON surestime en fait la précision de la méthode par coupe, en réduisant l'hétérogénéité des résultats, alors qu'avec la méthode visuelle, l'efficacité de l'observation est peu modifiée.

L'hypothèse d'une progression géométrique de la taille des colonies en fonction de la classe visuelle de l'arbre demanderait une vérification expérimentale, car elle a été introduite dans le modèle d'une façon très empirique.

Dans le cas de la méthode visuelle, les hypothèses constitutives du modèle sont beaucoup plus restreintes et concernent essentiellement la matrice de probabilités d'erreur. La dissymétrie de cette matrice introduit un biais puisque la sous-estimation de la classe est beaucoup plus fréquente que sa surestimation. Pour cette raison, il est important que l'étalonnage des indices ne soit pas fait à partir de classes réelles (en déterminant par exemple avec précision la classe d'une série d'arbres puis en effectuant un échantillonnage détaillé par coupe sur les mêmes arbres). La sous-estimation ultérieure serait alors systématique. Au contraire, l'étalonnage doit être fait en déterminant les classes dans les conditions courantes d'utilisation de la méthode : le risque de sous-estimation est ainsi inclus dans la détermination des effectifs des classes. Le problème de la variabilité du biais reste bien sûr posé. Néanmoins, les matrices de probabilités ayant un rôle assez limité dans la détermination de la précision d'échantillonnage, l'impact de ce biais est réduit.

\section{Distribution des résultats d'échantillonnage}

La distribution binomiale négative des effectifs moyens dans un verger, observée ici à l'issue du modèle de simulation par coupe de rameaux, concorde avec de nombreuses observations concernant des arthropodes nuisibles des plantes cultivées (in OGER, 1982). Le paramètre $\mathrm{K}$ de cette distribution est souvent considéré comme constant pour une espèce de ravageur et une culture données. TAYLOR et al. (1979) ont montré que ce paramètre dépendait en fait de la moyenne. Le modèle de simulation d'échantillonnage montre ici que $\mathrm{K}$ dépend non seulement de $\mathrm{N} 0, \mathrm{~N} 1$ et $\mathrm{U}$, qui définissent la moyenne, mais également de A, M0 et M1 qui définissent la pression d'échantillonnage ainsi que de $\mathrm{C} 0$ et $\mathrm{C} 1$ qui rendent compte des paramètres de distribution à l'intérieur de l'arbre. Dans le cas des populations de pucerons, la binomiale négative sert de base à l'élaboration de méthodes séquentielles en cultures annuelles (OGER, 1982) ; ce type d'approche pourrait donc être étendu aux plantes pérennes.

\section{Rôle des différents paramètres dans la précision des 2 méthodes}

L'impact des paramètres étudiés sur la précision des résultats d'échantillonnage est généralement différent selon la méthode que l'on utilise.

La raison $\mathrm{U}$ de la progression géométrique qui définit l'effectif d'un arbre d'une classe visuelle n'intervient pas dans le même sens avec la méthode par coupe de rameaux - où la dispersion des résultats est d'autant plus faible que l'effectif (donc U) est grand et avec la méthode visuelle - où une erreur de classe a d'autant plus d'importance que l'effectif est plus élevé. La valeur absolue de l'exposant de U (tabl. 4 et 6) est sensiblement supérieure avec la $1^{\text {re }}$ méthode. Ce paramètre y joue donc un rôle plus déterminant dans la définition de la précision d'échantillonnage.

Le nombre d'arbres échantillonnés intervient, lui, d'une manière similaire dans les 2 cas : il faut multiplier par 4 à 7 le nombre d'arbres examinés pour diviser par 2 le coefficient de variation. Le meilleur compromis entre la durée de l'opération, qui ne doit pas être trop importante, et le nombre de plantes examinées, qui doit être le plus grand possible, se situe autour d'une cinquantaine d'arbres.

La quantité de bois portée par les pêchers et sa variabilité influent peu sur la précision de la méthode par coupe de rameaux, sauf si le métrage est très faible, la qualité de l'estimation des populations s'améliorant alors fortement. Cependant, les rameaux coupés représentent dans ce cas une proportion importante du bois total et cette ablation, si elle est répétée, peut être préjudiciable à la plante (notamment pour de très jeunes 
arbres). Grossièrement, K0 est multiplié par 2 quand le métrage de rameaux est multiplié par 4 (ordre de grandeur de la croissance du bois pendant la saison de végétation).

La distribution des arbres dans les différentes classes visuelles est définie, pour un nombre d'arbres échantillonnés donné, par les paramètres No et N1 de la loi de POLYA-AEPPLI. La relation entre K0 (méthode par coupe de bois) et N0 est une fonction puissance négative alors que la liaison entre K1 (méthode visuelle) et No est linéaire. Pour une valeur de N1 égale à 10, et quand $\mathrm{N} 0$ passe de 2,5 à $9,5, \mathrm{~K} 0$ passe de 0,74 à 0,42 ; $\mathrm{K} 1$ passe de 0,73 à 0,43 avec une matrice d'erreur forte et de 0,70 à 0,59 avec une matrice d'erreur faible. L'amélioration de la précision d'échantillonnage avec le degré moyen d'infestation du verger est donc comparable pour les 2 méthodes (le parallélisme est du même ordre avec $\mathrm{N} 1=20$, en faisant varier $\mathrm{N} 0$ de 2,5 à 12,5). Dans le cas de contaminations très réduites (N0 inférieur à 2), nous avons vu que la dispersion des résultats de la méthode visuelle pouvait être très variable et dépendait de la prédominance d'arbres totalement sains (faible dispersion) ou d'arbres peu infestés (forte dispersion).

L'hétérogénéité de la répartition des pucerons à l'intérieur de la couronne des arbres influe sur le résultat de la méthode par coupe de rameaux : si la variation de la taille des colonies autour de leur moyenne (C1) n'a qu'un rôle très secondaire, il n'en est pas de même pour cette moyenne elle-même qui dépend du degré d'infestation de l'arbre : lorsque C0 est multiplié par 2, K0 est multiplié par 6 à 7. La précision de l'échantillonnage est donc beaucoup moins bonne lorsque les colonies sont de grande dimension, c'est-à-dire lorsqu'une grande quantité de pucerons est localisée à une portion restreinte de la couronne des arbres; le fait qu'une telle colonie soit ou non récupérée sur un rameau coupé pèse alors fortement sur le résultat moyen du verger.

Dans le cas de la méthode visuelle, la précision du résultat d'échantillonnage dépend assez peu de la matrice des probabilités d'erreur sauf en très faible densité (§ III.C.). Cette faiblesse de l'impact de la matrice peut sembler surprenante. La grande différence des 2 situations testées - erreur forte et erreur faible - confirme cependant ce résultat. En fait, compte-tenu de la définition des classes visuelles, le risque de se tromper de catégorie ne peut être que très faible pour une infestation suffisante (à partir de la classe 5 ou 6). En faible densité par contre, l'erreur peut être relativement importante.

Les paramètres de distribution des pucerons à l'intérieur de l'arbre (taille et répartition des colonies) ainsi que le métrage de rameaux ne peuvent s'exprimer, dans la méthode visuelle, que par l'intermédiaire de la matrice de probabilités. On peut donc conclure qu'ils y jouent un rôle beaucoup plus modéré que dans la méthode par coupe de bois, contribuant, avec la cou- leur des aphides et la nature des dégâts, à définir le degré de visibilité des espèces.

La hiérarchie des paramètres qui déterminent la précision de l'échantillonnage n'est donc pas la même pour les 2 méthodes : les raisons des 2 progressions géométriques, $\mathrm{U}$ et $\mathrm{C} 1$, sont les plus déterminantes pour la méthode de coupe de rameaux. Dans le cas de la méthode visuelle, si l'infestation est très faible, la précision dépend avant tout de la matrice de probabilités, donc du degré de visibilité de l'espèce. Si l'infestation est intermédiaire ou forte, la précision s'accroît avec le degré d'infestation (N0), quelle que soit l'espèce considérée, puisque les matrices d'erreur n'interviennent pratiquement plus et que $U$ (qui dépend également de l'espèce) doit être multiplié par 4 pour que le coefficient de variation de l'échantillonnage soit multiplié par 2 .

\section{CONCLUSION}

D'une façon générale, la méthode par coupe de rameaux est légèrement plus précise en faible densité, avec des espèces peu visibles, développant des colonies de faible dimension et bien réparties dans la couronne des arbres ( $M$. persicae). La méthode visuelle est par contre nettement plus efficace en forte densité, avec des espèces bien repérables, comportant des colonies de grande dimension ( $B$. persicae et $B$. prunicola). Le cas de 2 autres pucerons qui provoquent des dégâts importants sur pêchers, Hyalopterus pruni Geof. et Myzus varians David. reste à aborder.

En ce qui concerne la durée de mise en œuvre de l'échantillonnage, l'avantage est nettement à la méthode visuelle. Cette durée, pour les 2 méthodes, est sensiblement proportionnelle au nombre d'arbres examinés. Ce nombre influe, nous l'avons vu, de la même façon sur la précision des 2 techniques. Par contre, pour une cinquantaine d'arbres échantillonnés, l'examen visuel dure de 1 à $3 \mathrm{~h}$ selon la densité et l'état du couvert végétal alors qu'il faut $1 / 2 \mathrm{~h}$ de terrain mais 2 à $30 \mathrm{~h}$ de dépouillement au laboratoire (tri et comptages) pour la méthode par coupe de rameaux.

Le calcul de l'effectif de pucerons à l'ha nécessite par ailleurs la connaissance du métrage de bois d'un an moyen par arbre (afin de pouvoir rapporter le métrage échantillonné au métrage total). Dans le cas contraire, les populations ne peuvent être rapportées qu'au mètre de rameaux. La méthode visuelle ne nécessite aucune ablation d'organes et autorise donc des échantillonnages répétés, même sur de jeunes arbres. Enfin, elle permet, outre le calcul des effectifs de pucerons à l'ha, de suivre la dynamique des populations de chacun des arbres examinés, alors que la coupe de bois ne fournit qu'une information globale sur le verger.

Reçu le 22 mars 1984 Accepté le 19 octobre 1984. 


\section{RÉFÉRENCES BIBLIOGRAPHIQUES}

Anscombe F. J., 1949. The statistical analysis of insect counts based on the negative binomial distribution. Biometrics, 5, 165-173.

Baggiolini M., 1965. Méthode de contrôle visuel des infestations d'arthropodes ravageurs du pommier. Entomophaga, 10, 221-229.

Barideau L., Dagnélie P., Latteur G., 1975. Etude de l'échantillonnage de 3 espèces de pucerons des céréales. Bull. Rech. agron. Gembloux, 10, 3-12.

Lapchin L., Boll R., 1981. Utilisation de la typologie automatique pour la mise au point d'un échantillonnage stratifié, en vue de l'étude des relations plante hôte-pucerons en vergers de pêchers. Agronomie, I, 587-594.

Leclant F., Remaudière G., 1970. Elèments pour la prise en considération des aphides dans la lutte intégrée en vergers de pêchers. Entomophaga, 15, 53-81.
Oger R., 1982. Une procédure séquentielle pour le contrôle des maladies et des populations d'organismes nuisibles des plantes cultivees. Biom. Praxim., 22, 149-162.

Rabasse J.-M., Bouchery Y., 1977. Nouvelles données sur les méthodes d'évaluation des populations de pucerons (Homoptères, Aphididae) : séparation des insectes de leur plante hôte et dénombrement. Ann. Zool. Ecol. anim., 9, 407-423.

Tamaki G., 1973. Spring populations of the green peach aphid on peach trees and the role of natural enemies in their control. Environ. Entomol., 2, 186-191.

Taylor L. R., Woiwod I. P., Perry J. N., 1979. The negative binomial as a dynamic ecological model for aggregation, and the density dependence of k. J. anim. Ecol., 48, 289-304.

Waters T. F., 1969. Subsampler for dividing large samples of stream invertebrate drift. Limnol. Oceanogr., 14, 813-815. 